\title{
Das Prostatakarzinom - Innovationsschub in Diagnostik und Therapie!
}

$\mathrm{D}$ as Prostatakarzinom gilt heute insgesamt als der häufigste maligne Tumor des Mannes und hat damit in seiner Häufigkeit das Bronchialkarzinom überholt. Allein in Europa müssen wir jährlich mit etwa 2,6 Millionen neuen Fällen rechnen. Außerdem ist das Prostatakarzinom für ungefähr $10 \%$ aller karzinombedingten Todesfälle verantwortlich. Zahlen wie diese machen die enorme sozioökonomische Bedeutung dieses Tumors deutlich. Da das Prostatakarzinom ein Krebs des älteren Mannes ist, wird, insbesondere vor dem Hintergrund der sich verändernden demografischen Struktur in einem Land wie Deutschland evident, wie wichtig die Rolle von Diagnostik und Behandlung dieses Tumors in der Medizin ist.

Wurden noch vor 20 Jahren in den meisten Fällen erst fortgeschrittene Tumore mit dann insgesamt doch eher ungünstiger Prognose diagnostiziert und behandelt, so änderte sich mit der Einführung moderner diagnostischer Verfahren das klinische Bild. Heute entdecken wir Prostatakarzinome meist zu einem Zeitpunkt, zu dem eine hohe Wahrscheinlichkeit besteht, diese Erkrankung kurativ behandeln zu können. Wesentlich hierfür ist die geradezu revolutionierende Einführung des prostataspezifischen Antigens (PSA) in die Diagnostik des Prostatakarzinoms. Das typische, heute entdeckte Prostatakarzinom ist ein im Rahmen einer Vorsorgeuntersuchung entdeckter kurativ zu behandelnder, asymptomatischer Tumor.

Die drei Säulen der Prostatakarzinomdiagnostik (digital-rektale Untersuchung, PSA, transrektal sonografiegeführte Biopsie), die Küfer dem Nicht-Urologen in diesem Heft nahe bringt - sollten demnach einen festen Platz im Kopf eines jeden klinisch tätigen Mediziners haben. Er fokussiert dabei bewusst auf die wesentlichen Punkte der modernen Prostatadiagnostik, ohne dezidiert auf die neuesten Entwicklungen im urologischen Fachgebiet einzugehen. So können die Möglichkeiten der differenzierten PSA-Diagnostik, teilweise unterstützt durch Computersimulationen, wie auch aktuelle Entwicklungen in der Bildgebung (PET-CT, Magnetresonanztomografie), trotz ihres großen Potenzials im Gefüge dieses Heftes keine Berücksichtigung finden.

Auch das therapeutische Vorgehen bei der Diagnose „Prostatakarzinom“ hat sich in den letzten 20 Jahren deutlich gewandelt. So galt früher die operative Behandlung des Prostatakarzinoms im Regelfall als ein heroischer, mit großen Risiken behafteter Eingriff, der lediglich an wenigen Zentren halbwegs sicher durchgeführt werden konnte. Heute ist die Technik der radikalen Prostatektomie in den meisten urologischen Hauptabteilungen ein Routineeingriff.

Die Fortentwicklung der operativen Verfahren stellen Hautmann Jr. und Simon dar, die aufzeigen, dass in der heutigen Zeit vor allem Faktoren, welche die Lebens-

qualität des Patienten (Potenz!) und den Wunsch nach einem minimalinvasiven Vorgehen (Laparoskopie) berücksichtigen, eine wichtige Rolle spielen. Dies gilt natürlich genauso für die moderne Strahlentherapie des Prostatakarzinoms, deren Möglichkeiten Messer in diesem Heft beschreibt. Ausgeklügelte Bestrahlungsplanungssysteme und neuartige Bestrahlungstechniken (IMRT, Brachytherapie) haben den Strahlentherapeuten einen gleichermaßen ausgeprägten Innovationsschub in der Behandlung des Prostatakarzinoms verschafft, wie dem operativ tätigen Urologen.

Heute führen in der Behandlung des lokal begrenzten Prostatakarzinoms mehrere Wege zum gleichen Ziel. Daher sind wir auch immer besser in der Lage, auf individuelle Wünsche und Vorstellungen des Patienten einzugehen. Immer klarer scheint zu werden, dass beim Prostatakarzinom eine spezifische Prävention möglich ist. So lassen epidemiologische Studien (die Wahrscheinlichkeit eines Afroamerikaners, an einem Prostatakarzinom zu erkranken, ist 30-mal größer als die eines Japaners) eine präventive Diätetik vermuten. Inwieweit sich dies für bestimmte Nahrungsergänzungssubstanzen (Vitamin E, Selen) wirklich nachweisen lässt, bleibt derzeit noch spekulativ. Dasselbe gilt für eine mögliche medikamentöse Prävention, unter Verwendung von Substanzen, die in den Androgenstoffwechsel eingreifen (5-alphaReduktasehemmer).

Das zeigt uns wieder einmal, mit welcher Rasanz sich der medizinische Kenntnisstand beim Prostatakarzinom entwickelt. In wenigen Jahren wird vieles von dem, was heute Standard und wohl belegtes Vorgehen ist, genauso zum alten Eisen gehören, wie wir es für so manch anderes beim Prostatakarzinom in den letzten 20 Jahren erlebt haben. Deshalb muss der heute klinisch tätige Arzt über dieses wichtige Krankheitsbild „Prostatakarzinom“ im Interesse seiner Patienten ein Grundwissen erwerben. Ziel der Beiträge über das Prostatakarzinom ist es, Ihnen dieses Grundwissen näher zu bringen und Sie damit in die Lage zu versetzen, Ihre betroffenen Patienten in die richtigen Bahnen zu führen - zum Urologen!

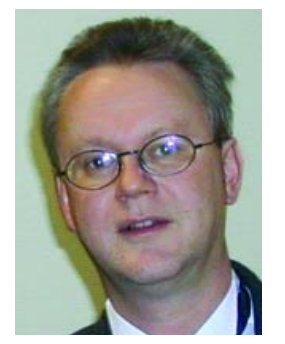

PD Dr. H.-W. Gottfried, UIm

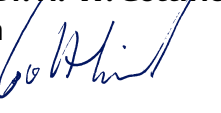

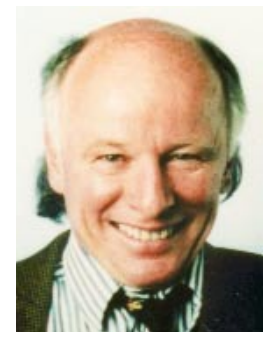

Prof. Dr. R.E. Hautmann, Ulm (Gasteditor)

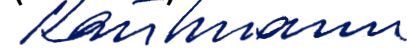

